\title{
Dual antibody immunohistochemistry: an efficient and sensitive tool for the detection of residual disease in chronic lymphocytic leukemia
}

\author{
Laura M Wake ${ }^{1}$ (D) Inhye E Ahn ${ }^{2} \cdot$ Mohammed Z. Farooqui $^{2} \cdot$ Xin Tian $^{3} \cdot$ Maryalice Stetler-Stevenson $^{4} \cdot$ \\ Gerald E. Marti ${ }^{2} \cdot$ Adrian Wiestner $^{2} \cdot$ Irina Maric $^{5}$
}

Received: 13 June 2019 / Accepted: 11 September 2019/Published online: 8 November 2019

(C) Springer-Verlag GmbH Germany, part of Springer Nature 2019

\begin{abstract}
Highly effective treatments for chronic lymphocytic leukemia (CLL) have the potential to reduce significant tumor burden to single cells and therefore require sensitive tools to assess for minimal residual disease (MRD) in bone marrow (BM) biopsies. Flow cytometry (FC) is the current gold standard for detection of MRD, but requires a specialized facility, specific antibody panels, collecting hundreds of thousands-to-millions of cells, and personnel with expertise in the analysis and interpretation of FC MRD data, which may not be feasible in many small laboratories. Dual-antibody immunohistochemistry (DA-IHC) can identify abnormal populations better than morphology alone, but its correlation with FC assessment is not known. Our aims are to characterize the efficacy of DA-IHC in assessing BM samples post-treatment and to compare results with FC to evaluate for residual disease in CLL. We collected 2-year posttherapy data from 33 CLL patients on two treatment protocols, chemoimmunotherapy (CIT) and single agent ibrutinib (IB), as well as BMs from 10 healthy volunteers as morphologic controls. BM biopsy specimens were examined for the presence or absence of CLL based on morphologic evidence of lymphoid infiltration and aberrant co-expression of CD5 and PAX5 DA-IHC. FC using a standard CLL antibody panel was performed in parallel. All IB patients had residual disease detected by DA-IHC and FC, although five patients (22\%) were morphologically negative by routine hematoxylin and eosin (H\&E) stain. Those without overt morphologic evidence of disease showed DA-IHC-positive interstitial single cells and were positive for residual disease by FC. The assessments by DA-IHC and FC were significantly correlated $(p=0.004)$. Four patients $(40 \%)$ treated with CIT were morphologically negative by H\&E, and two of these had no detectable CLL by either DA-IHC or FC. The other two patients had low-level disease detected by both DA-IHC and FC. The MRD levels identified by DA-IHC were correlated with those by FC $(p<0.0001)$. We characterized the efficacy of DA-IHC in assessing CLL post-treatment and found assessments by DA-IHC and FC were statistically significant in both the IB- and CIT-treated patients. These findings suggest DA-IHC could accurately detect residual disease in cases lacking morphologic evidence by H\&E alone. DA-IHC may be a useful tool in current practice as another sensitive and efficient method to assess for MRD in CLL.
\end{abstract}

Electronic supplementary material The online version of this article (https://doi.org/10.1007/s12308-019-00372-w) contains supplementary material, which is available to authorized users.

\section{Laura M Wake}

Lwake1@jhmi.edu

1 Pathology Department, Johns Hopkins Medicine, Weinberg 2321, 410 N. Broadway, Baltimore, MD 21231, USA

2 Hematology Branch, National Heart, Lung and Blood Institute, National Institutes of Health, Bethesda, MD, USA

3 Office of Biostatistics Research, National Heart, Lung, and Blood Institute, National Institutes of Health, Bethesda, MD, USA

4 Laboratory of Pathology, National Cancer Institute, National Institutes of Health, Bethesda, MD, USA

5 Department of Laboratory Medicine, National Institutes of Health, Bethesda, MD, USA
Keywords Dual-antibody immunohistochemistry · Chronic lymphocytic leukemia · Minimal residual disease

\section{Introduction}

Significant recent advances in understanding tumor biology have led to discoveries of novel treatment options for patients with chronic lymphocytic leukemia (CLL) [1]. Current highly effective treatment options have the potential to reduce heavy CLL tumor burden to single circulating cells. Due to these dramatic treatment responses, effective monitoring of residual disease, which may impact prognosis and potential future treatment decisions, requires more sophisticated and sensitive assays, and must also be technically feasible in all hospital laboratories.

Per the International Workshop on Chronic Lymphocytic Leukemia Working Group (IWCLL-WG) 2018 guidelines, 
several diagnostic tools should be employed to assess the efficacy of a given CLL treatment, as defined by complete remission (CR), partial remission (PR), progressive disease (PD), and stable disease (SD), including morphologic evaluation of the peripheral blood and bone marrow [1]. For patients in clinical trials with apparent CR, the IWCLL-WG recommends assessing for minimal residual disease (MRD), which is defined as 1 CLL cell in 10,000 cells, using standardized technologies, including 6-color flow cytometry, allele-specific oligonucleotide PCR, or high-throughput sequencing using the ClonoSEQ assay [1]. MRD assessment can be performed on the peripheral blood (PB), unless the patient has been treated with antibody-based regimens, because those therapies preferentially clear the blood but not the bone marrow (BM) of disease. In those cases, assessment of the BM for MRD is required [1]. Not surprisingly, microscopic evaluation of a BM with low-level disease is difficult based on routine hematoxylin and eosin (H\&E)-stained slides alone, and therefore, the IWCLL-WG recommends the use of more sensitive assays, such as flow cytometry (FC) or molecular tests in assessing for MRD [1-4]. Immunohistochemistry (IHC) can assist pathologists evaluating the bone marrow, but morphologic assessment using single-antibody IHC (SA-IHC) is not as sensitive as FC [3]. However, MRD FC testing has its own challenges and special requirements [4], including a specialized facility, specific antibody panels, and the collection of hundreds of thousands-to-millions of cells, not to mention the experience and expertise of a pathologist trained in MRD testing, which is more complex than other diagnostic FC assays, all of which may not be feasible in many laboratories.

Novel automated dual-antibody immunohistochemistry (DA-IHC) can identify aberrant co-expression of tumor cells with high sensitivity. DA-IHC can identify abnormal populations better than morphology alone [5-8], but its correlation with $\mathrm{FC}$ assessment in assessing residual disease is not known. Our aims are to characterize the efficacy of DA-IHC in assessing CLL burden post-treatment with different treatment protocols and to compare results with FC, a gold standard in the evaluation of MRD.

\section{Materials and methods}

We collected data from 33 CLL patients on two different treatment protocols at our institution. The patients, 21 men and 12 women, were between 36 and 85 years of age, with a median age of 67 years. The patients were grouped based on their treatment protocols: 10 patients treated with 3 cycles of chemoimmunotherapy (CIT) consisting of fludarabine plus ofatumumab with or without cyclophosphamide, and 23 patients treated with continued daily dosing of single agent ibrutinib (IB). Patients on the CIT arm were treatment-naïve before protocol enrollment at our institution, while the patients on the IB arm had previously diagnosed relapsed and/or refractory disease or high-risk characteristics prior to protocol enrollment (Supplemental Table 1).

Two-year post-therapy clinical and laboratory data was recorded, and BM aspirate and biopsy procedures were performed at our institution on all 33 patients. A physical examination was performed, and a complete blood count (CBC) and white blood cell (WBC) differential were recorded on the date of the BM biopsy. In addition, as a control, BM biopsies were obtained from 10 healthy patient volunteers with no history of CLL, and with normal CBCs and other laboratory parameters.

BM biopsy aspirates were collected in EDTA, and a predetermined portion was submitted for both FC and cytogenetic analysis. Aspirate smears were made at bedside and later stained with Wright-Giemsa. Core biopsies were processed in our laboratory in the usual fashion, with formalin fixation followed by decalcification, paraffin-embedding, and sectioning at $4 \mathrm{uM}$. Routine $\mathrm{H} \& \mathrm{E}$-stained slides were prepared and core biopsies were stained with CD20 (Ventana Medical Systems, Inc., Tucson, AZ), CD3 (Ventana Medical Systems, Inc., Tucson, AZ), and CD5 (Ventana Medical Systems, Inc., Tucson, AZ). In addition, core biopsies were dual-stained with antibodies against CD5, a red membranous and cytoplasmic stain, and PAX5, a brown nuclear stain (Ventana Medical Systems, Inc., Tucson, AZ). All immunohistochemical stains were performed via automated Ventana Benchmark Ultra stainers as per the manufacturer's protocols using standard procedures. All staining protocols were specifically validated on decalcified bone marrow samples.

All 33 patient core biopsies and 10 control biopsies were microscopically examined by two hematopathologists and scored based on (1) the presence or absence of lymphoid aggregates or infiltration and the extent of involvement as identified by H\&E-stained slides, and (2) the presence or absence of dual-positive B cells, and the location and extent of involvement. Lymphoid aggregates, defined as $>15$ clustered lymphocytes, were designated as absent/negative ( 0 ) or present/positive $(\geq 1)$. The number of $\mathrm{B}$ cells expressing aberrant CD5/PAX5 DA-IHC was compared with the total B cells present and recorded as negative ( $0 \%$ of all $\mathrm{B}$ cells), rare $(>0$ to $<10 \%$ of all $\mathrm{B}$ cells), moderate ( $\geq 10$ to $<50 \%$ of all $\mathrm{B}$ cells), or extensive ( $\geq 50 \%$ of B cells) (Table 1$)$. In the case of normal controls, BM core biopsies were examined for the number of dual-positive B cells per 10 high-power fields (HPF) $(\times 40)$. Single antibody immunohistochemical (SAIHC) staining with CD20 and CD5 and CD3 was recorded on all cases for comparison, but these cases were not blinded and the results were not included in the statistics.

FC assessment for MRD in BM aspirates was performed on all 33 CLL patients at our facility. Specimens were processed within $12 \mathrm{~h}$ of collection. Whole blood lysis was performed using ammonium chloride prior to staining 
Table 1 Left—BM findings in patients treated with chemoimmunotherapy (CIT). Right—BM findings in patients treated with ibrutinib (IB)

\begin{tabular}{|c|c|c|c|}
\hline Pt & $\begin{array}{c}\text { Morphology } \\
\text { H\&E }\end{array}$ & $\begin{array}{c}\text { DA-IHC } \\
\text { B-cells }\end{array}$ & $\begin{array}{c}\text { FC } \% \text { Clonal } \\
\text { B-cells / total } \\
\text { lymphs; B-cells }\end{array}$ \\
\hline CIT1 & Negative & $\geq 0-<10 \%$ & $1 \% ; 10 \%$ \\
\hline CIT2 & Positive & $\geq 50 \%$ & $87 \% ; 99 \%$ \\
\hline CIT3 & Positive & $\geq 50 \%$ & $56 \% ; 91 \%$ \\
\hline CIT4 & Negative & $0 \%$ & $0 \%$ \\
\hline CIT5 & Negative & $\geq 0-<10 \%$ & $0.5 \% ; 1 \%$ \\
\hline CIT6 & Positive & $\geq 0-<10 \%$ & $10 \% ; 17 \%$ \\
\hline CIT7 & Positive & $\geq 10-<50 \%$ & $22 \% ; 36 \%$ \\
\hline CIT8 & Positive & $\geq 50 \%$ & $61 \% ; 99 \%$ \\
\hline CIT9 & Positive & $\geq 50 \%$ & $74 \% ; 98 \%$ \\
\hline CIT & Negative & $0 \%$ & $0 \%$ \\
\hline 10 & & & \\
\hline
\end{tabular}

\begin{tabular}{|c|c|c|c|}
\hline Pt & $\begin{array}{c}\text { Morphology } \\
\text { H\&E }\end{array}$ & $\begin{array}{c}\text { DA-IHC } \\
\text { B-cells }\end{array}$ & $\begin{array}{c}\text { FC } \% \text { Clonal } \\
\text { B-cells /total } \\
\text { Iymphs; B-cells }\end{array}$ \\
\hline IB1 & Positive & $\geq 50 \%$ & $73 \% ;>99 \%$ \\
\hline IB2 & Negative & $\geq 0-<10 \%$ & $2 \% ; 99 \%$ \\
\hline IB3 & Positive & $\geq 50 \%$ & $53 \% ; 99 \%$ \\
\hline IB4 & Positive & $\geq 50 \%$ & $58 \% ;>99 \%$ \\
\hline IB5 & Positive & $\geq 50 \%$ & $53 \% ; 89 \%$ \\
\hline IB6 & Positive & $\geq 50 \%$ & $35 \% ; 91 \%$ \\
\hline IB7 & Positive & $\geq 10-<50 \%$ & $25 \% ; 74 \%$ \\
\hline IB8 & Positive & $\geq 10-<50 \%$ & $9 \% ; 82.3 \%$ \\
\hline IB9 & Positive & $\geq 50 \%$ & $33 \% ;>99 \%$ \\
\hline IB10 & Positive & $\geq 50 \%$ & $58 \% ; 99 \%$ \\
\hline IB11 & Negative & $\geq 10-<50 \%$ & $2 \% ; 87 \%$ \\
\hline IB12 & Negative & $\geq 0-<10 \%$ & $0.8 \% ; 14 \%$ \\
\hline IB13 & Negative & $\geq 0-<10 \%$ & $13 \% ; 44 \%$ \\
\hline IB14 & Positive & $\geq 50 \%$ & $58 \% ;>99 \%$ \\
\hline IB15 & Positive & $\geq 50 \%$ & $49 \% ; 98 \%$ \\
\hline IB16 & Positive & $\geq 50 \%$ & $72 \% ;>99 \%$ \\
\hline IB17 & Positive & $\geq 50 \%$ & $57 \% ; 95 \%$ \\
\hline IB18 & Negative & $\geq 50 \%$ & $58 \% ; 99 \%$ \\
\hline IB19 & Positive & $\geq 50 \%$ & $58 \% ; 97 \%$ \\
\hline IB20 & Positive & $\geq 50 \%$ & $46 \% ; 93 \%$ \\
\hline IB21 & Positive & $\geq 50 \%$ & $83 \% ; 99 \%$ \\
\hline Positive & $\geq 50 \%$ & $65 \% ; 97.6 \%$ \\
\hline Positive & $\geq 50 \%$ & $32 \% ; 92 \%$ \\
\hline
\end{tabular}

Morphology $\mathrm{H} \& \mathrm{E}=$ negative or positive disease based on morphology alone. DA-IHC $=\%$ of B cells positive for dual-antibody immunohistochemistry. Second column $=$ pink $=$ positive morphology; blue $=$ negative morphology. Third column $=$ pink $\geq 50 \%$ of B cells are DA-IHC-positive; light blue $=0$ $10 \%$ of B cells are positive for DA-IHC; dark blue $=\geq 10-<50 \%$ of B cells are DA-IHC-positive. Fourth column $=$ FC $\%$ clonal B cells/total lymphocytes; FC $\%$ clonal B-cells/all B cells; pink $=>50 \%$ of B cells are clonal by FC; dark blue $=>0<50 \%$ of B cells are clonal; light blue $=0 \%$ clonal B cells detected by FC

for $30 \mathrm{~min}$ at room temperature with an eight-color cocktail (antibody concentration per manufacturer's recommendations) containing antibodies to CD19, CD20, CD22, CD79b, CD5, CD23, CD81, CD43, CD45, CD3, CD13, CD14, CD16, and CD56, and surface kappa and lambda light chains. At least 1 million cells were acquired per tube using an 8-color multiparametric approach on a 3laser FACS Canto II (BD Biosciences, San Jose, CA) with DiVa 6.1.1 software and analyzed by FCS Express 4 software (DeNovo Software, Los Angeles, CA). The limit of detection of this method is $0.002 \%$. Assessment for MRD was performed by pathologists with expertise in FC. Gating on CD19-positive events, any discreet cluster of 3 or more events with light-chain restriction, dim CD20, and aberrant CD5 was deemed residual disease.

Descriptive statistics were performed. The percentages of clonal B cells determined by DA-IHC were scored as $0,1,2$, and 3 for negative, rare, moderate, and extensive, and Spearman's rank correlation $\rho$ between the percentages of clonal B cells determined by DA-IHC and flow cytometry was calculated. All tests were two-sided, and a $p$ value $<$ 0.05 was considered statistically significant. 


\section{Results}

All patients were assessed for clinical response at 2 years from the start of the study treatment by physical examination and laboratory studies, including $\mathrm{CBC}$ and $\mathrm{WBC}$ differential count (Supplemental Table 2). In the IB treatment group $(n=23)$, three patients $(13 \%)$ had lymphocytosis above $4 \times$ 1000/microL. Fifteen patients $(65 \%)$ had cytopenias, seven patients $(30 \%)$ had palpable lymphadenopathy, and four (17\%) had either hepatomegaly and/or splenomegaly. Fluorescence in situ hybridization (FISH) and/or cytogenetic results showed three patients $(13 \%)$ with trisomy 12 , four patients (17\%) with del13q, five patients $(22 \%)$ with del11q, and eleven patients $(48 \%)$ with del17p. Molecular studies showed mutated IGHV in ten patients $(43 \%)$ and unmutated IGHV in thirteen patients (57\%) (Supplemental Table 1).

Patients treated with CIT $(n=10)$ had normal-to-low lymphocyte counts, and six (60\%) had cytopenias, most often mild thrombocytopenia. Five patients (50\%) had palpable lymphadenopathy, and none ( $0 \%)$ had hepatosplenomegaly. FISH and/or cytogenetics revealed that four patients (40\%) had normal cytogenetics, three patients (30\%) had trisomy 12 , two patients $(20 \%)$ had del13q, one patient $(10 \%)$ had del11q, and no patients $(0 \%)$ had del17p. Molecular analysis of nine patients showed that only one patient $(11 \%)$ had mutated IGHV, while eight patients $(89 \%)$ were unmutated (Supplemental Table 1).

For patients treated with IB, all patients (100\%) had residual disease in the bone marrow as detected by DA-IHC and flow cytometry, although five patients $(22 \%)$ had morphologically undetectable disease by $\mathrm{H} \& \mathrm{E}$ alone (Fig. 1). SA-IHC identified only rare interstitial B cells, and aberrant co-expression of SA-IHC B cell markers and T cells markers was difficult to assess. For example, patient IB11 had no lymphoid aggregates or lymphocytosis detected on H\&E, but DA-IHC PAX5/CD5 showed scattered clustered interstitial cells $(\sim 5$ cells) with dual-positivity (Fig. 2a), and FC showed $87 \%$ of all B cells were clonal ( $2 \%$ of all lymphocytes). Patient IB7 had scattered lymphoid aggregates and a mild lymphocytosis on H\&E. SA-IHC was not helpful, as it showed the lymphoid aggregates were composed of a mixture of B cells and T cells, a finding which may be seen in normal reactive BM lymphoid aggregates, and which by itself, is not evidence of residual CLL. Most interstitial cells were positive for either CD20 (normal B cells) or for CD5 (normal T cells), but it was difficult to assess co-expression of CD20 and CD5. However, scattered cells were dual-positive with DA-IHC, indicating that a subset of the population represented residual CLL (Fig. 2b), and FC showed clonal B cells comprised 74\% of all B cells ( $25 \%$ of all lymphocytes). Interestingly, patient IB18 had no definitive morphologic evidence of disease by H\&E, but a subtle lymphocytosis was apparent with SA-IHC and DA-IHC, and FC showed $99 \%$ of all B cells were clonal (58\% of total lymphocytes). In fact, DA-IHC revealed that all patients on IB had involved BM, and the extent of involvement was significantly correlated with extent of involvement by FC (Spearman's correlation $\rho=0.58, p=0.004$, Fig. 1).

Of the five IB patients that were called negative based on H\&E and SA-IHC, all eventually went off study between 44 and 72 months; three of the patients remained in response to

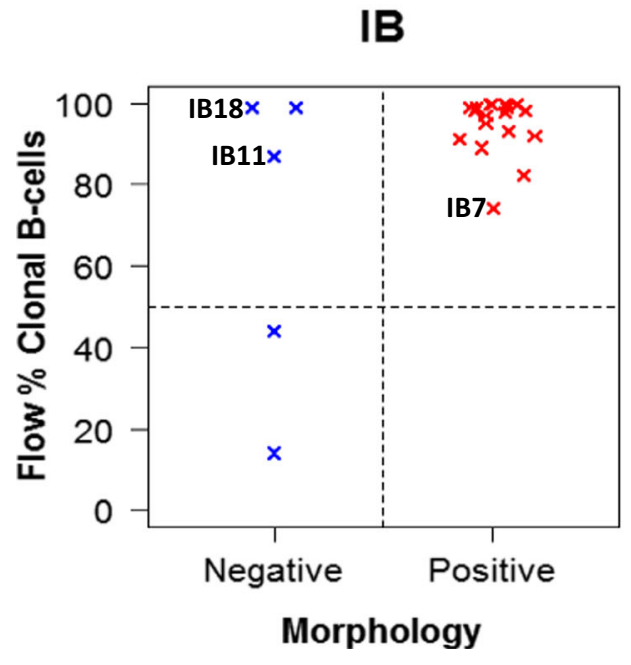

Fig. 1 Left-morphologic (H\&E) vs. FC examination of BM in patients treated with IB. Five patients are morphologically negative for disease by H\&E. Red = positive morphology (lymphocytosis or large lymphoid nodules $)$. Blue $=$ negative morphology. Right - DA-IHC vs. FC in patients treated with IB. All patients have residual disease, detected by
IB

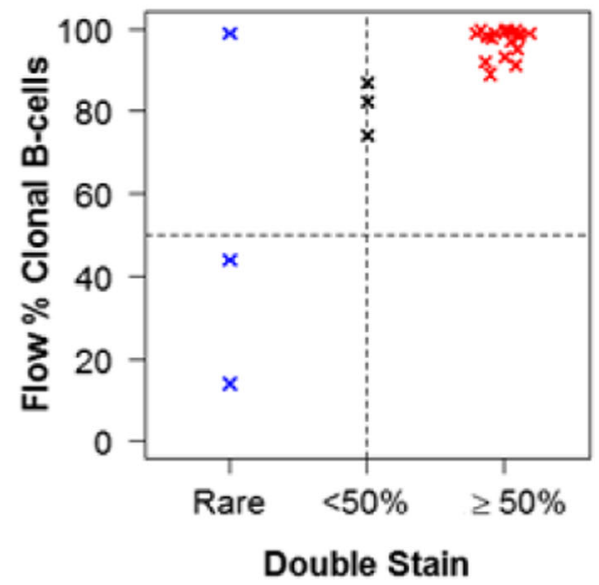

both DA-IHC and FC. Patients with fewer clonal B cells by FC have only rare dual-positive cells. Red $=>50 \%$ of B cells are DA-IHC-positive. Black $=<50 \%$ of B cells are DA-IHC-positive. Blue $=$ rare B cells are DA-IHC-positive 
Fig. 2 a Bone marrow core biopsy. No definitive

morphologic evidence of CLL by $\mathrm{H} \& \mathrm{E}$ in patient IB11 (top- $\mathrm{H} \& \mathrm{E}$ $\times 60$ ). PAX5/CD5 shows scattered clustered dual-positive cells at the arrow (bottomPAX5/CD5 $\times 60$ ). b Bone marrow core biopsy. H\&E and $\mathrm{PAX} 5 / \mathrm{CD} 5$ showing residual CLL in patient IB7 (top- $\mathrm{H} \& \mathrm{E} \times$ 60 ; bottom-PAX5/CD5 $\times 60$; inset $-\mathrm{PAX} 5 / \mathrm{CD} 5 \times 100$ )
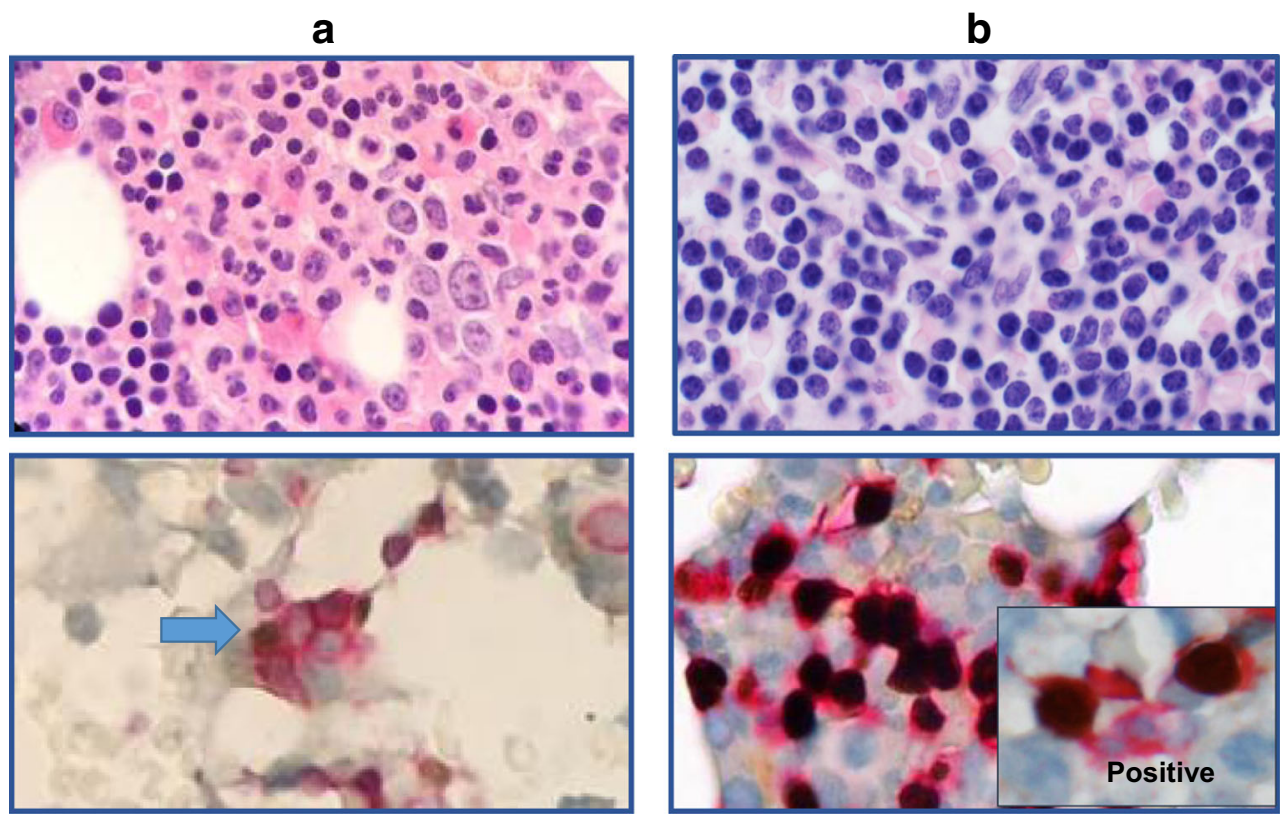

ibrutinib, but developed other malignancies (IB2, IB11, and IB18); one remained in response but developed recurrent pleural effusions related to Ibrutinib (IB12); and one remained in response for 72 months, when he developed progressive CLL and went off study (IB13).

For patients treated with CIT, four out of ten patients (40\%) had no overt morphologic evidence of disease on H\&E, and two of ten (20\%) patients had no detectable CLL by either DA-IHC or FC (Fig. 3). Patient CIT4 showed only scattered $\mathrm{B}$ cells and T cells, with no dual-positive cells, and no clonal B cells by FC (Fig. 4a). Two patients, including patient CIT5, were morphologically negative on $\mathrm{H} \& \mathrm{E}$, but had rare dualpositive interstitial cells (Fig. 4b). Most patients with morphologically identifiable disease had extensive DA-IHC-positive $\mathrm{B}$ cells ( $\geq 50 \%$ of all $\mathrm{B}$ cells) and in 15 out of 16 of these cases, clonal B cells comprised $>90 \%$ of all B cells detected by FC (Table 1). Correlation between the DA-IHC scores and the percentage of clonal B cells by FC was strong and statistically significant (Spearman's $\rho=0.96, p<0.0001$, Fig. 3).

The normal volunteer BMs were also assessed for dualpositive B cells: Five BMs (50\%) had no dual-positive B cells detected in 10 HPF. Four out of the other five patients (40\%) had $<5$ dual-positive cells in $10 \mathrm{HPF}$, and one patient (10\%) had 9 dual-positive cells in 10 HPF. Dual-positive B cells were distributed singly, with no clustering, and none of the BMs showed clusters $>3-5$ B cells together, which differs from the clustered dual-positive B cells seen in residual CLL. While there is no standard cutoff for the acceptable number of normal B cells expressing CD5, any cluster $>3-5$ CD5-positive $B$ cells was considered abnormal. Normal volunteer BM samples were not submitted for FC evaluation.

\section{Discussion}

Treatment strategies revolutionized by recent and ongoing academic investigations have not only improved the quality of life for many patients with CLL but have potentiated sustainable long-term responses, thus generating a need for more sensitive assays to detect low-level disease. Although a bone marrow biopsy is not typically necessary for the primary diagnosis of CLL in general practice, it is necessary to assess for evidence of MRD in apparent $\mathrm{CR}$, which is currently one of the best indicators for progression-free survival (PFS) in CLL [9-11].

Historically, pathologists examined the BM for residual disease based on morphologic assessment for lymphoid aggregates or lymphoid infiltration on H\&E-stained slides. To increase diagnostic sensitivity, current recommendations include the use of molecular methods or FC. Although not used in current clinical practice, high throughput sequencing using ClonoSEQ assays have been shown to have good concordance with FC MRD results [1], and this technique may play a significant role to complement MRD FC in the future.

IHC antibodies for B cell antigens (CD20, CD79a, and/or PAX5) and T cell antigens (CD3 and CD5) are helpful in the morphologic evaluation of BMs and are readily available in most pathology laboratories. However, methods of estimating CD20/CD79a/PAX5 and CD5 aberrant co-expression using SA-IHC are not precise, and while a small cluster $(\sim 5$ cells or less) of CD20-positive B cells is suspicious in a patient with a history of CLL, a definitive diagnosis of residual CLL is difficult and highly subjective, even amongst hematopathologists, especially when admixed $\mathrm{T}$ cells are present; detecting aberrant co-expression on low-level, single-cell CLL involvement is 
Fig. 3 Left -morphology (H\&E) vs. FC in patients treated with CIT. Four patients appear negative by H\&E alone. Red = positive morphology

(lymphocytosis or large lymphoid aggregates). Blue $=$ negative morphology. Right-DA-IHC vs. $\mathrm{FC}$ in patients treated with CIT. Both DA-IHC and FC show only two patients are negative for residual disease. The correlation between \% clonal B cells by FC and by DA-IHC is statistically significant. Red $=>50 \%$ of B cells are double-positive. Black $=$ $<50 \%$ of B cells are doublepositive. Blue $=$ rare $\mathrm{B}$ cells are double-positive. Green = no double-positive cells detected

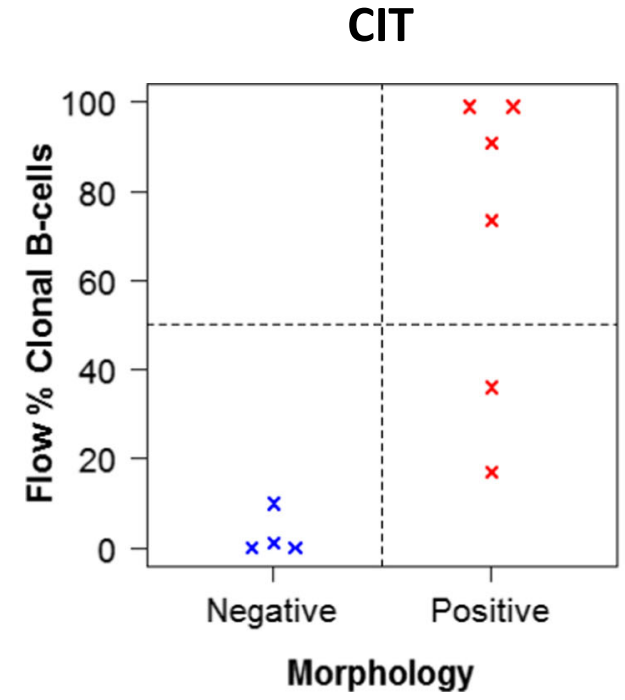

CIT

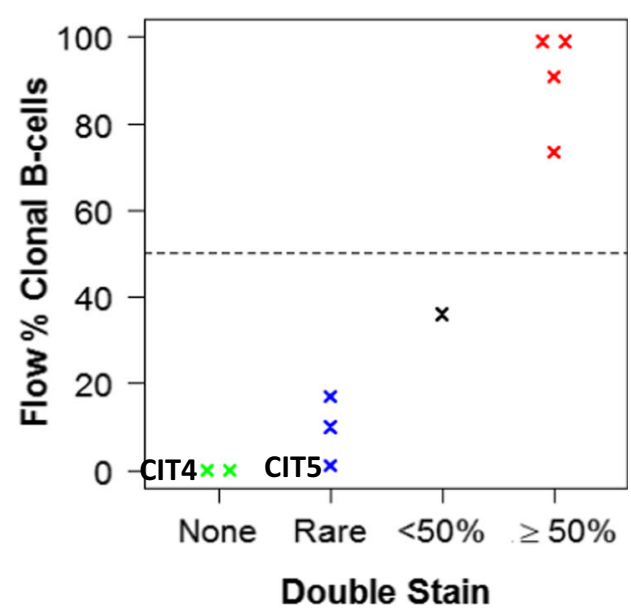

exceedingly difficult using only SA-IHC. Using DA-IHC techniques, we assessed residual disease in 33 patients with CLL under two different treatment protocols and compared the findings with FC.

In patients treated with $\mathrm{IB}, \mathrm{H} \& \mathrm{E}$ and $\mathrm{SA}-\mathrm{IHC}$ detected residual disease in $18 / 23$ cases $(78 \%)$, DA-IHC detected dual-positive cells in all cases (100\%), and FC showed positivity in all cases (100\%). In addition, the degree of involvement was similar; patients with rare B cell positive with DAIHC $(\geq 0<10 \%)$ had $<20 \%$ clonal B cells/total lymphocytes detected by FC, and those with $\geq 50 \%$ positive $\mathrm{B}$ cells by DAIHC had $>80 \%$ clonal B cells/total lymphocytes detected by FC. These patients had high-risk markers, such as age $>$ 65 years, deletion $17 \mathrm{p}$, and unmutated IGHV, and had all previously been treated and had failed conventional therapy at outside hospitals, thus prompting their enrollment in the IB protocol at our institution, a clinical trial intentionally enrolling high-risk patients. For this reason, therefore, these patients have a greater chance of residual disease involvement at the end of treatment, an assumption borne out by the findings.

Patients on the CIT protocol were previously untreated, and their distribution of prognostic markers (Rai stage, FISH, IGHV mutation status) were typical for treatmentnaïve CLL $[12,13]$. There were four patients with no morphologic evidence of residual disease by H\&E. However, two of these patients had residual disease detected by DA-IHC and FC, while two were truly negative by both DA-IHC and FC. The results between DA-IHC and FC showed strong and
Fig. 4 a Bone marrow core biopsy. No definitive

morphologic evidence of CLL by $\mathrm{H} \& \mathrm{E}$ in patient CIT4 (top- $\mathrm{H} \& \mathrm{E}$ $\times 60)$. DA-IHC confirms no disease (bottom-PAX5/CD5 $\times$ 60; inset-PAX5/CD5 × 100). b Bone marrow core biopsy. H\&E shows scattered lymphocytes in patient CIT5. DA-IHC confirms a few small clusters of dual-positive cells at the arrow (top- $\mathrm{H} \& \mathrm{E} \times$ 60; bottom-PAX5/CD5 $\times 60$ ) a

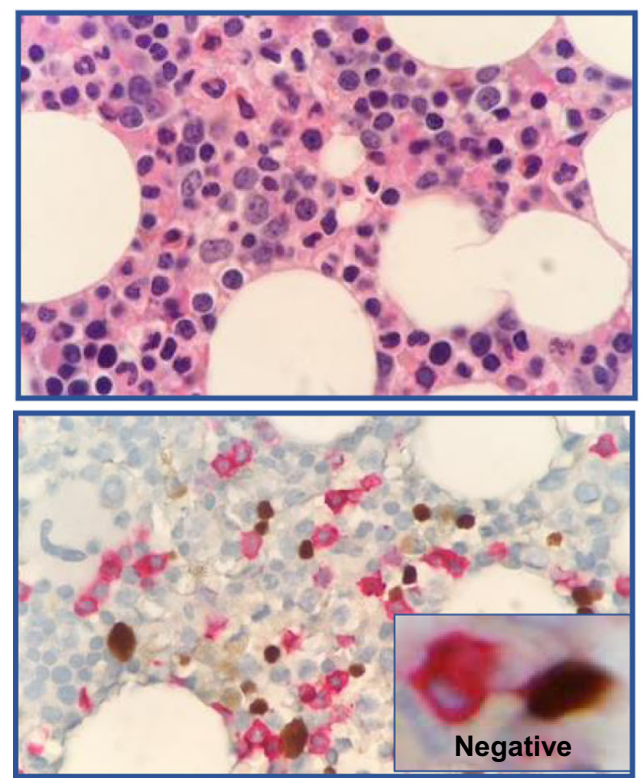

b
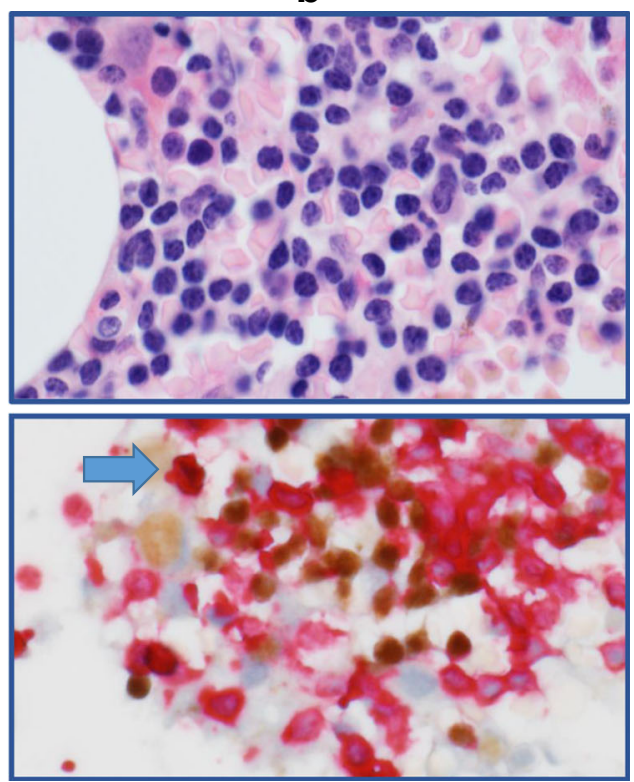
positive correlation for both treatment groups ( $\rho=0.58, p=$ 0.004 and $\rho=0.96, p<0.0001$, respectively).

The distinction between a negative and a positive result is important for patients undergoing treatment. Complete response to treatment, as defined by FC MRD negativity, is an independent predictor of progression-free survival (PFS) and overall survival (OS) in patients treated with chemotherapy or chemoimmunotherapy, even when compared with IGHV mutational status, ZAP70 status, TP53 defects, and cytogenetic risk factors $[9,14-17]$ and may be used to identify candidates for treatment-dose de-escalation [16]. It is thought to be a superior risk calculator as compared with other prognostic markers, as the response to treatment takes into account all variables, including tumor biology, as well as host characteristics, such as patient metabolism and patient compliance [14].

These survival studies use FC to assess MRD, and the significance of detecting a group of 5 cells with DA-IHC in regard to patient survival, at this point, is unknown. However, based on our data, detection of DA-IHC-positive cells indicated residual disease detectable by FC.

It is important to morphologically assess a BM with negative FC results because there is a potential for discrepancies. One study that examined the detection of CLL by MRD FC vs. morphologic assessment found 5 out of $77 \mathrm{BM}$ were falsely negative by FC [4]. Three of these cases had morphologically low-level disease, one had focal areas of diffuse infiltration, and one was extensively involved. In these cases, the differences between morphology and FC were thought to be due to sampling error. It is also advisable to perform morphologic review of BMs with the aid of IHC. Clusters of five or more CD20-positive B cells, or anything greater than scattered positive $\mathrm{B}$ cells in the $\mathrm{BM}$, are suspicious in a patient with a history of CLL; the expertise of a hematopathologist or utilizing a consensus plan may be recommended to make a diagnosis in these situations, but it is also necessary to use additional IHC to confirm that the B cells are CLL cells, and not normal regenerating B cells [3]. While normal B cells may coexpress CD5 and PAX5, as we recorded in 5/10 normal control BMs, clusters of such cells in patients with a history of CLL indicate residual disease. However, as previously mentioned, in cases of very low-level disease, it may be difficult to determine aberrant expression of $\mathrm{CD} 5$ on $\mathrm{B}$ cells using SAIHC alone.

To our knowledge, this is the first reported comparison between DA-IHC and FC for residual disease detection in patients with CLL. We characterized the efficacy of DA-IHC in assessing CLL post-treatment and found statistically significant correlation between DA-IHC and FC assessment results. Although sensitive methods such as FC and molecular tests are irreplaceable for the confirmation of MRD, DA-IHC may provide a useful tool in current practice as another sensitive and cost-effective method to assess for residual disease in CLL patients.

\section{Compliance with ethical standards}

Conflict of interest Pharmacyclics and Novartis provided study drug and research support for the study. M.F. is employed at Merck \& Co., Inc., Kenilworth, NJ, USA; owns stocks; and has received travel support from Merck. M.F. was an employee of the National Institutes of Health, Bethesda, MD, at the time of this study. A.W. received research support from Pharmacyclics, LLC, an AbbVie Company. Other authors declare that they have no conflict of interest.

\section{References}

1. Hallek M, Cheson BD, Catovsky D, Caligaris-Cappio F, Dighiero $\mathrm{G}$, Dohner $\mathrm{H}$ et al (2018) iwCLL guidelines for diagnosis, indications for treatment, response assessment, and supportive management of CLL. Blood. 131(25):2745-2760

2. Hallek M, Cheson BD, Catovsky D, Caligaris-Cappio F, Dighiero G, Dohner H et al (2008) Guidelines for the diagnosis and treatment of chronic lymphocytic leukemia: a report from the International Workshop on Chronic Lymphocytic Leukemia updating the National Cancer Institute-Working Group 1996 guidelines. Blood. 111(12):5446-5456

3. Rawstron AC, Kennedy B, Evans PA, Davies FE, Richards SJ, Haynes AP et al (2001) Quantitation of minimal disease levels in chronic lymphocytic leukemia using a sensitive flow cytometric assay improves the prediction of outcome and can be used to optimize therapy. Blood. 98(1):29-35

4. Rawstron AC, Villamor N, Ritgen M, Bottcher S, Ghia P, Zehnder JL et al (2007) International standardized approach for flow cytometric residual disease monitoring in chronic lymphocytic leukaemia. Leukemia. 21(5):956-964

5. Hudnall SD, Ge Y, Wei L, Yang N-P, Wang H-Q, Chen T (2005) Distribution and phenotype of Epstein-Barr virus-infected cells in human pharyngeal tonsils. Mod Pathol 18(4):519-527

6. Khokhar FA, Payne WD, Talwalkar SS, Jorgensen JL, BuesoRamos CE, Medeiros LJ, Vega F (2010) Angioimmunoblastic Tcell lymphoma in bone marrow: a morphologic and immunophenotypic study. Hum Pathol 41(1):79-87

7. Masir N, Jones M, Lee AM, Goff LK, Clear AJ, Lister A, Marafioti T, Mason DY (2010) The expression of Bcl-2 by proliferating cells varies in different categories of B-cell lymphoma. Histopathology. 56(5):617-626

8. Rimsza LM, Day WA, McGinn S, Pedata A, Natkunam Y, Warnke R, Cook JR, Marafioti T, Grogan TM (2014) Kappa and lambda light chain mRNA in situ hybridization compared to flow cytometry and immunohistochemistry in B cell lymphomas. Diagn Pathol 9:144

9. Bottcher S, Ritgen M, Fischer K, Stilgenbauer S, Busch RM, Fingerle-Rowson G et al (2012) Minimal residual disease quantification is an independent predictor of progression-free and overall survival in chronic lymphocytic leukemia: a multivariate analysis from the randomized GCLLSG CLL8 trial. J Clin Oncol : Off J Am Soc Clin Oncol 30(9):980-988

10. Santacruz R, Villamor N, Aymerich M, Martinez-Trillos A, Lopez C, Navarro A, Rozman M, Bea S, Royo C, Cazorla M, Colomer D, Gine E, Pinyol M, Puente XS, Lopez-Otin C, Campo E, Lopez-Guillermo A, Delgado J (2014) The prognostic impact of minimal residual disease in patients with chronic lymphocytic leukemia requiring first-line therapy. Haematologica. 99(5):873-880

11. Kwok M, Rawstron AC, Varghese A, Evans PA, O’Connor SJ, Doughty $\mathrm{C}$ et al (2016) Minimal residual disease is an independent 
predictor for 10-year progression-free and overall survival in CLL. Blood. 128:2770-2773

12. Hallek M, Fischer K, Fingerle-Rowson G, Fink AM, Busch R, Mayer J, Hensel M, Hopfinger G, Hess G, von Grünhagen U, Bergmann M, Catalano J, Zinzani PL, Caligaris-Cappio F, Seymour JF, Berrebi A, Jäger U, Cazin B, Trneny M, Westermann A, Wendtner CM, Eichhorst BF, Staib P, Bühler A, Winkler D, Zenz T, Böttcher S, Ritgen M, Mendila M, Kneba M, Döhner H, Stilgenbauer S (2010) Addition of rituximab to fludarabine and cyclophosphamide in patients with chronic lymphocytic leukaemia: a randomised, open-label, phase 3 trial. Lancet. 376(9747):1164-1174

13. Eichhorst B, Fink AM, Bahlo J, Busch R, Kovacs G, Maurer C, Lange E, Köppler H, Kiehl M, Sökler M, Schlag R, Vehling-Kaiser U, Köchling G, Plöger C, Gregor M, Plesner T, Trneny M, Fischer K, Döhner H, Kneba M, Wendtner CM, Klapper W, Kreuzer KA, Stilgenbauer S, Böttcher S, Hallek M, international group of investigators, German CLL Study Group (GCLLSG) (2016) First-line chemoimmunotherapy with bendamustine and rituximab versus fludarabine, cyclophosphamide, and rituximab in patients with advanced chronic lymphocytic leukaemia (CLL10): an international, open-label, randomised, phase 3, non-inferiority trial. Lancet Oncol 17(7):928-942
14. Böttcher S (2014) Paving the road to MRD-guided treatment in CLL. Blood. 123(24):3683-3684

15. Goede V, Fischer K, Busch R, Engelke A, Eichhorst B, Wendtner CM, Chagorova T, de la Serna J, Dilhuydy MS, Illmer T, Opat S, Owen CJ, Samoylova O, Kreuzer KA, Stilgenbauer S, Döhner H, Langerak AW, Ritgen M, Kneba M, Asikanius E, Humphrey K, Wenger M, Hallek M (2014) Obinutuzumab plus chlorambucil in patients with CLL and coexisting conditions. N Engl J Med 370(12):1101-1110

16. Strati P, Keating MJ, O'Brien SM, Burger J, Ferrajoli A, Jain N, Tambaro FP, Estrov Z, Jorgensen J, Challagundla P, Faderl SH, Wierda WG (2014) Eradication of bone marrow minimal residual disease may prompt early treatment discontinuation in CLL. Blood. 123(24):3727-3732

17. Molica S, Giannarelli D, Montserrat E (2019) Minimal residual disease and survival outcomes in patients with chronic lymphocytic leukemia: a systematic review and meta-analysis. Clin Lymphoma, Myeloma Leuk 19(7):423-430

Publisher's note Springer Nature remains neutral with regard to jurisdictional claims in published maps and institutional affiliations. 